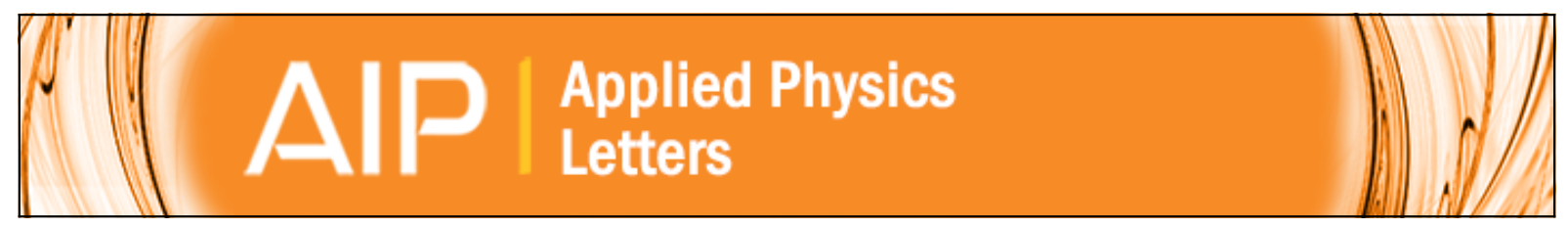

\title{
Controlling magnetic vortices through exchange bias
}

J. Sort, G. Salazar-Alvarez, M. D. Baró, B. Dieny, A. Hoffmann, V. Novosad, and J. Nogués

Citation: Applied Physics Letters 88, 042502 (2006); doi: 10.1063/1.2165290

View online: http://dx.doi.org/10.1063/1.2165290

View Table of Contents: http://scitation.aip.org/content/aip/journal/apl/88/4?ver=pdfcov

Published by the AIP Publishing

\section{AlP Re-register for Table of Content Alerts}

\section{Create a profile. \\ Sign up today!}




\title{
Controlling magnetic vortices through exchange bias
}

\author{
J. Sort ${ }^{\mathrm{a})}$ \\ Institució Catalana de Recerca i Estudis Avançats (ICREA) and Departament de Física, Universitat \\ Autònoma de Barcelona, 08193 Bellaterra, Barcelona, Spain \\ G. Salazar-Alvarez and M. D. Baró \\ Departament de Física, Universitat Autònoma de Barcelona, 08193 Bellaterra, Spain \\ B. Dieny \\ SPINTEC (URA 2512 CNRS/CEA), CEA/Grenoble, 17 Avenue Martyrs, 38054 Grenoble Cedex 9, France
}

\begin{abstract}
A. Hoffmann and V. Novosad
Materials Science Division and Center for Nanoscale Materials, Argonne National Laboratory, Argonne, Illinois 60439

\section{J. Nogués}

Institució Catalana de Recerca i Estudis Avançats (ICREA) and Departament de Física, Universitat Autònoma de Barcelona, 08193 Bellaterra, Barcelona, Spain
\end{abstract}

(Received 11 November 2005; accepted 18 December 2005; published online 23 January 2006)

\begin{abstract}
The magnetization reversal in Permalloy (Py) and Py-IrMn disks ( $1 \mu \mathrm{m}$ diameter) is investigated. The Py disks reverse their magnetization via vortex state formation. Conversely, if the Py-IrMn disks are field cooled from above the blocking temperature $T_{B}$, a critical angle with respect to the cooling direction is set, beyond which the vortex no longer nucleates. This angle can be experimentally tuned by varying the magnitude of the exchange bias field. Furthermore, the coupling with IrMn can also induce an enhancement of the vortex stability when the disks are zero-field cooled from above $T_{B}$. C 2006 American Institute of Physics. [DOI: 10.1063/1.2165290]
\end{abstract}

The study of magnetization reversal in patterned magnetic structures is interesting for both fundamental and technological reasons. ${ }^{1,2}$ When the size of the magnetic element becomes comparable to critical length scales, such as the domain wall width, the multidomain structure of the bulk becomes energetically unfavorable and either single-domain or inhomogeneous magnetization configurations are preferred. ${ }^{3-7}$ Specifically, in circular magnetic dots, as the magnetic field is reduced from saturation, the magnetization tends to curl up along the edges of the nanostructures to minimize the lateral stray fields, leading to a flux closure (i.e., vortex) arrangement. The vortex formation results in a sudden drop of the magnetization at the so-called nucleation field. As the applied field is changed to negative saturation, the core of this vortex moves perpendicularly to the field direction until it is expelled from the dot at the so-called annihilation field.

It is noteworthy that although the influence of dot shape, size, and interdot distance on magnetization reversal has been extensively investigated in homogeneous magnetic systems, ${ }^{8-12}$ limited work has been carried out so far on how possible interlayer exchange coupling effects can influence the magnetization reversal in patterned elements. One example would be the ferromagnetic (FM)-antiferromagnetic (AFM) exchange coupled structures. ${ }^{13}$ These systems typically exhibit a shift of the hysteresis loop, along the magnetic field axis, referred to as the exchange bias field $\left(H_{E}\right)$, which has found applications in many magnetotransport devices. $^{14-17}$

The effects arising from the competition between magnetostatic and exchange bias energies in rings, and circular or

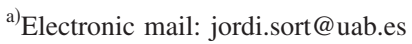

elliptical dots are controversial. Although some authors report a suppression of the flux-closure states, ${ }^{18,19}$ others clearly show that vortex configurations are possible in exchange biased systems. ${ }^{20-22}$ Furthermore, some studies on submicron exchange biased rings show complex reversal mechanisms with asymmetric hysteresis loops. ${ }^{23,24}$ Recently, we have demonstrated that circular Py-IrMn nanostructures can exhibit either reversal via an exchange biased vortex or coherent rotation, depending on the measuring angle. ${ }^{20}$

In this letter, we demonstrate that the thermomagnetic history in exchange biased disks determines their magnetization reversal mechanisms and, in fact, this reversal can be controlled by subjecting the disks to different heat treatments. When the disks are zero-field cooled (ZFC) from above the blocking temperature $\left(T_{B}\right)$ of the system, that is, the temperature at which exchange bias effects vanish upon heating, both the nucleation and annihilation fields of the vortex increase with respect to unbiased Permalloy ${ }^{\mathrm{TM}}$ (Py) disks, resulting in an enhancement of the magnetic field range within which the vortex state persists. In contrast, if the FM-AFM disks are cooled under the presence of saturating magnetic fields, a tunable critical angle can be introduced, beyond which the vortex state no longer nucleates.

A continuous film with the composition $\mathrm{Ta}(5 \mathrm{~nm}) /$ Py $(12 \mathrm{~nm}) / \operatorname{IrMn}(5 \mathrm{~nm}) / \mathrm{Pt}(2 \mathrm{~nm})$ (where Py stands for $\mathrm{Ni}_{80} \mathrm{Fe}_{20}$ and is FM, whereas IrMn, i.e., $\mathrm{Ir}_{20} \mathrm{Mn}_{80}$, is AFM) was deposited onto a thermally oxidized Si wafer by dc magnetron sputtering. For comparison, a sample with composition Ta $(5 \mathrm{~nm}) / \mathrm{Py}(12 \mathrm{~nm}) / \mathrm{Pt}(2 \mathrm{~nm})$, was also prepared. From the continuous films, arrays of circular disks with diameter of $1 \mu \mathrm{m}$ and periodicity $2 \mu \mathrm{m}$ were fabricated by e-beam lithography and subsequent ion etching. These structures were then subjected to different types of heat treatment 

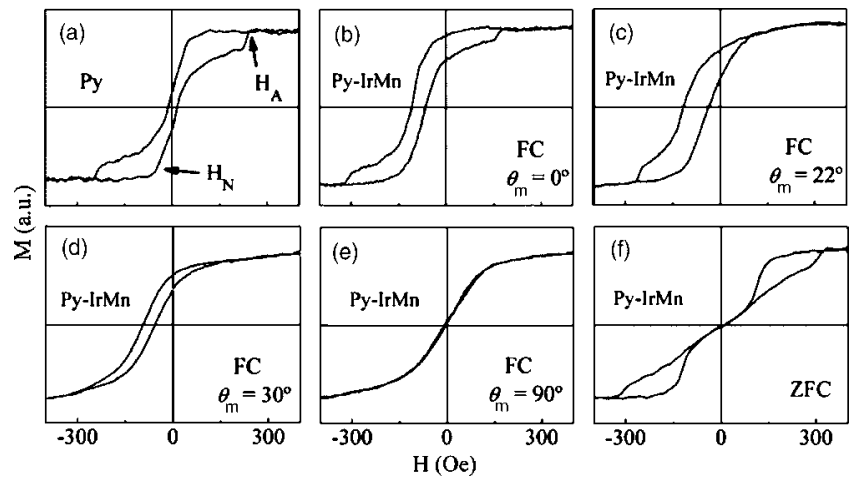

FIG. 1. Hysteresis loops, measured at room temperature of (a) the Py disks, (b)-(e) the Py-IrMn disks after FC from $550 \mathrm{~K}$ and measured along several in-plane angles $\left(\theta_{m}=0^{\circ}, 22^{\circ}, 30^{\circ}\right.$ and $\left.90^{\circ}\right)$, and (f) the Py-IrMn disks measured after ZFC from $550 \mathrm{~K}$.

procedures: (i) heating to $550 \mathrm{~K}$, which is above $T_{B},{ }^{25}$ and subsequently field cooling (FC) to room temperature in a $H_{\mathrm{FC}}=+2.5 \mathrm{kOe}$ in-plane magnetic field; (ii) heating to $550 \mathrm{~K}$, ac degaussing and zero-field cooling to room temperature; and (iii) to tune the magnitude of the exchange bias field, the samples initially FC in a positive field were subsequently heated again but to a lower temperature $\left(T_{A}<T_{B}\right)$ and cooled to room temperature in a negative field: $H_{\mathrm{FC}}=$ $-2.5 \mathrm{kOe}$. Hysteresis loops were measured at room temperature with fields applied at various in-plane angles $\theta_{\mathrm{m}}$ from the FC direction, using a Durham Magneto Optics Kerr effect setup.

The hysteresis loop corresponding to the Py disks (without the AFM) is shown in Fig. 1(a). The loop shape is typical for magnetization reversal via nucleation and annihilation of a vortex state. The nucleation and annihilation fields are $H_{N}=35 \mathrm{Oe}$ and $H_{A}=245 \mathrm{Oe}$, respectively. The loop shape (and also $H_{N}$ and $H_{A}$ ) are insensitive to the direction of measurement. It should be noted that the reversal via vortex state formation is what is expected for the diameter and thickness of these disks. ${ }^{3,9}$

Shown in Figs. 1(b)-1(e) are the hysteresis loops of the Py-IrMn disks after FC from $550 \mathrm{~K}$ to room temperature, measured along several in-plane angles from the FC direction, which defines $0^{\circ}$. As can be seen in Fig. 1(b), the loop recorded along $0^{\circ}$ resembles that of Py disks but is now shifted along the magnetic field axis by $H_{E}=88 \mathrm{Oe}$. After recentering the loop for the exchange bias field, one obtains values for $H_{N}$ and $H_{A}$ very similar to those of the unbiased Py disks. As the measuring angle is increased, a slight decrease in $H_{N}$ and $H_{A}$ is observed, which is accompanied with the appearance of an asymmetry in the loop shape [see Fig. 1 (c)]. For angles larger than about $22^{\circ}$ no traces of the vortex state are visible, and the loops suggest reversal by coherent rotation [Figs. 1(d) and 1(e)].

In exchange biased 400-nm-diameter Py-IrMn circular dots, we have recently reported the existence of a critical angle $\left(\theta_{C}\right)$, beyond which the vortex state no longer nucleates. ${ }^{20}$ Indeed, as a first approximation, one can describe the coupling with the AFM as an additional field (i.e., $H_{E, 0}$, the exchange bias field measured along $0^{\circ}$ ) superimposed to the applied external field. Along $0^{\circ}, H_{E, 0}$ can be effectively compensated by the external field, thus allowing the Py-IrMn disks to reverse via a shifted vortex state. However, if $H_{E, 0}$ is exceedingly large compared to $H_{N_{0}}$ (the
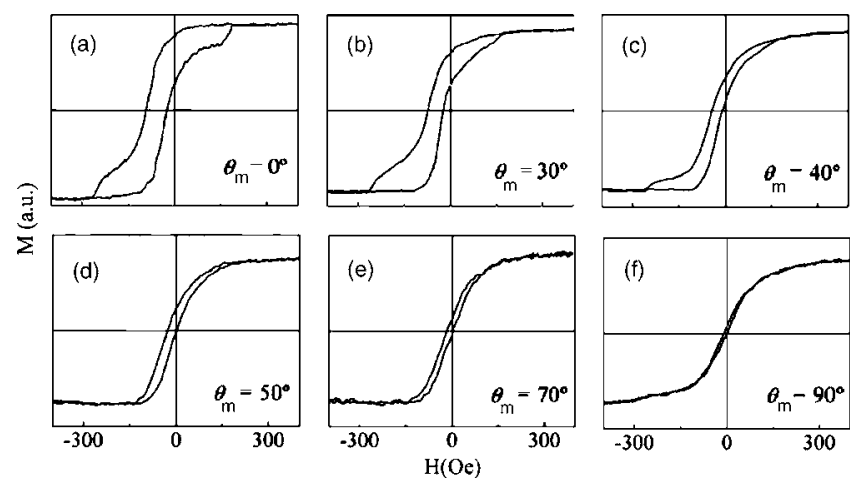

FIG. 2. Hysteresis loops of the Py-IrMn disks measured along various inplane angles $\left(\theta_{m}=0^{\circ}, 30^{\circ}, 40^{\circ}, 50^{\circ}, 70^{\circ}\right.$, and $\left.90^{\circ}\right)$ after field cooling from $550 \mathrm{~K}$ using a positive field, $H_{\mathrm{FC}}=2.5 \mathrm{kOe}$, and subsequently field cooling again but now using $H_{\mathrm{FC}}=-2.5 \mathrm{kOe}$ from $T_{A}=350 \mathrm{~K}$.

nucleation field for $H$ along $0^{\circ}$ ) then, beyond a critical angle, the external field is no longer sufficiently large to compensate $H_{E, 0}$ and, as a result, the vortex state does not nucleate. Using a simple geometrical argument, it can be shown that ${ }^{20}$

$$
\theta_{C}=\arcsin \left(\frac{H_{N, 0}}{H_{E, 0}}\right)
$$

From the values of $H_{N, 0}$ and $H_{E, 0}$ for the $1 \mu \mathrm{m}$ dots one obtains a critical angle of around $22^{\circ}$, in agreement with the experimental observations. In the 400-nm-diameter Py-IrMn structures $H_{N, 0}$ was larger and $H_{E, 0}$ smaller and thus $\theta_{C}$ was much larger than for the $1 \mu \mathrm{m}$ disks. ${ }^{20}$

From this description, it can be envisaged that the value of $\theta_{C}$ might be controllably varied if one could tune either $H_{N, 0}$ or $H_{E, 0}$ (or both). Although $H_{N}$ is, a priori, dependent only on the geometry of the dots (i.e., diameter and thickness) and it is thus constant for a given sample, such a tuning of $H_{E, 0}$ can, in principle, be indeed carried out by combining FC processes using fields of opposite sign. ${ }^{25,26}$ Namely, once the array of dots has been FC from $550 \mathrm{~K}$ using a positive field, $H_{E, 0}$ can then be subsequently decreased by heating again the sample to a temperature $\left(T_{A}\right)$ below $T_{B}$ and then cooling it using a negative field. This allows pinning different parts of the AFM along opposite directions and, as a result, the net exchange bias tends to average out; that is, gradually decreases as $T_{A}$ increases. Taking advantage from the fact that after the first FC $\theta_{C}$ of the $1 \mu \mathrm{m}$ dots is rather small, we have carried out this second FC under a negative field with the aim of controllably increasing $\theta_{C}$. As an example, shown in Fig. 2 are the hysteresis loops measured along different in-plane angles after cooling the disks in a negative field from $T_{A}=350 \mathrm{~K}$. This second FC procedure decreases $H_{E, 0}$ to $56 \mathrm{Oe}$ and, remarkably, $\theta_{C}$ consequently increases to around $40^{\circ}$. In addition, an asymmetric loop shape is again observed around the critical angle, which may be ascribed to the delicate energy balance at $\theta_{C}$ between reversal via vortex nucleation and coherent rotation.

By progressively increasing $T_{A}, H_{E, 0}$ decreases and $\theta_{C}$ increases, while the average $H_{N}$ and $H_{A}$ fields for $\theta_{m}=0^{\circ}$ remain constant. Such dependences of $H_{E, 0}$ and $\theta_{C}$ on $T_{A}$ are shown in Figs. 3(a) and 3(b). In addition, plotted as inset is the dependence of $\sin \left(\theta_{C}\right)$ on $1 / H_{E, 0}$. It is observed that a linear relationship exists between $\sin \left(\theta_{C}\right)$ on $1 / H_{E, 0}$, as expected from Eq. (1), taking into account that the $H_{N, 0}$ remains constant for the various experiments. Furthermore, 


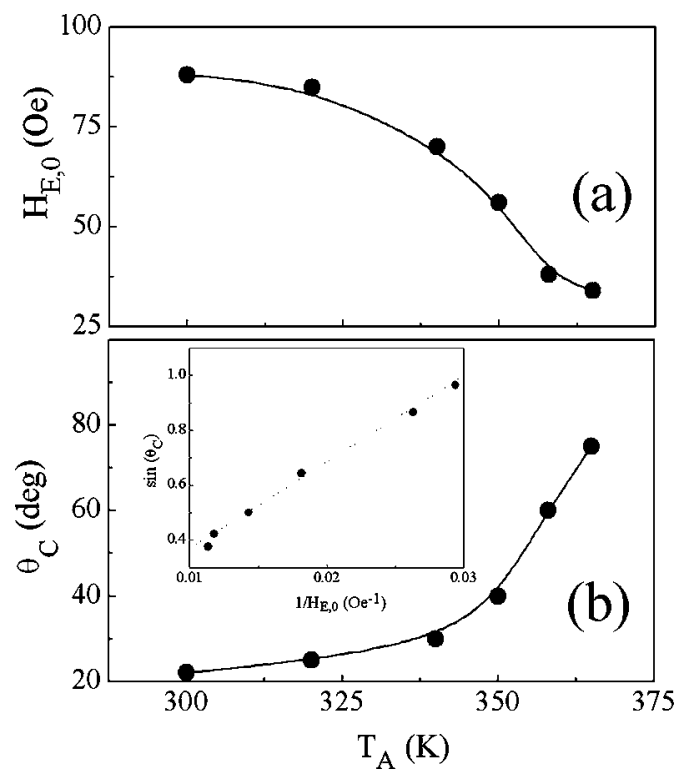

FIG. 3. (a) Dependence of $H_{E, 0}$ on the second field cooling (i.e., using $H_{\mathrm{FC}}=-2.5 \mathrm{kOe}$ ) temperature $T_{A}$, (b) dependence of $\theta_{C}$ on $T_{A}$, and as inset, dependence of $\sin \left(\theta_{C}\right)$ on $1 / H_{E, 0}$. The continuous lines in (a) and (b) are guides to the eye, whereas the discontinuous line in the inset is a linear fit of the data.

from the slope of the fit, a value of 33 Oe is obtained, which approximately corresponds to the nucleation field of the vortex along $0^{\circ}$ once the loop has been recentered [Fig. 1(b)].

The Py-IrMn disks have also been ZFC from $550 \mathrm{~K}$. The resulting hysteresis loop measured at room temperature is shown in Fig. 1(f). It is remarkable that the disks again reverse via vortex state for any value of the angle of measurement (without appreciable angular dependence). However, both $H_{N}$ and $H_{A}$ now increase with respect to the unbiased Py disks or the FC Py-IrMn disks. This means that during the hysteresis loop, starting from saturation, the vortex state nucleates at a higher decreasing field and annihilates at a larger opposite increasing field than in Py disks, indicating an enhanced magnetic stability of the vortex state. Note also that the vortex deformation when a field is applied between the nucleation field and annihilation field is much more reversible than in simple Py disks or after FC.

Interestingly enough, the enhancement of both $H_{N}$ and $H_{A}$ is of about $90 \mathrm{Oe}$; that is, the enhancement is identical to the $H_{E, 0}$ value obtained after FC the Py-IrMn disks from $550 \mathrm{~K}$. This suggests that during the ZFC process, the vortex state has been imprinted into the AFM and that, once at room temperature, the coupling with the AFM acts like a 90 Oe local effective pinning field of cylindrical symmetry.

Finally, it should be noted that the fact that it is possible to tune $H_{E}$ by combining two $\mathrm{FC}$ using fields of opposite sign means that, actually, the AFM layer is composed of a certain number of subunits (most likely, regions of correlated uncompensated spins), whose orientation can be modified so as to be either parallel or antiparallel to $0^{\circ}$ (in the case of the FC treatments) ${ }^{27}$ or following a vortex-like configuration (as suggested by the loops measured after ZFC).

In conclusion, it has been shown that the magnetization reversal mechanisms in Py disks can be modified in a controlled manner by exchange coupling the Py with an AFM layer, without the need of varying the disks thickness or diameter. Depending on the thermomagnetic history of the sample, these disks can reverse via vortex state or by coherent rotation. Actually, after subjecting them to a field-cooling process in a saturating field from above the blocking temperature, a critical angle is found to be set, beyond which reversal occurs by coherent rotation. This angle can be experimentally tuned by adjusting the value of the exchange bias field. Furthermore, if the Py-IrMn disks are subjected to a zero-field cooling process, the stability of the vortex state is enhanced, in the sense that both the nucleation and annihilation fields increase with respect to unbiased Py disks.

Financial support from the NEXBIAS (HPRN-CT 200200296), the 2005SGR-00401, the MAT-2004-01679 research projects and the Institut Català de Nanotecnologia (ICN) is acknowledged. Work at Argonne was supported by the U.S. Department of Energy, under Contract No. W-31-109-ENG38. One of the authors (G.S.A.) acknowledges the financial support from the NEXBIAS research network.

${ }^{1}$ J. I. Martín, J. Nogués, K. Liu, J. L. Vincent, and I. K. Schuller, J. Magn. Magn. Mater. 256, 449 (2003).

${ }^{2}$ R. Skomski, J. Phys.: Condens. Matter 15, R841 (2003).

${ }^{3}$ R. P. Cowburn, D. K. Koltsov, A. O. Adeyeye, M. E. Welland, and D. M. Tricker, Phys. Rev. Lett. 83, 1042 (1999).

${ }^{4}$ K. Yu. Guslienko, V. Novosad, Y. Otani, H. Shima, and K. Fukamichi, Phys. Rev. B 65, 024414 (2001).

${ }^{5}$ J. K. Ha, R. Hertel, and J. Kirschner, Phys. Rev. B 67, 224432 (2003).

${ }^{6}$ X. Liu, J. N. Chapman, S. McVitie, and C. D. W. Wilkinson, J. Appl. Phys. 96, 5173 (2004).

${ }^{7}$ P. Vavassori, N. Zaluzec, V. Metlushko, V. Novosad, B. Ilic, and M. Grimsditch, Phys. Rev. B 69, 214404 (2004).

${ }^{8}$ P.-O. Jubert and R. Allenspach, Phys. Rev. B 70, 144402 (2004).

${ }^{9}$ H. Hoffmann and F. Steinbauer, J. Appl. Phys. 92, 5463 (2002).

${ }^{10}$ M. Natali, I. L. Prejbeanu, A. Lebib, L. D. Buda, K. Ounadjela, and Y. Chen, Phys. Rev. Lett. 88, 157203 (2002).

${ }^{11}$ V. Novosad, K. Yu. Guslienko, H. Shima, Y. Otani, S. G. Kim, K. Fukamichi, N. Kikuchi, O. Kitakami, and Y. Shimada, Phys. Rev. B 65, 060402(R) (2002).

${ }^{12}$ J. Shi, S. Tehrani, and M. R. Scheinfein, Appl. Phys. Lett. 76, 2588 (2000).

${ }^{13}$ J. Nogués, J. Sort, V. Langlais, V. Skumryev, S. Suriñach, J. S. Muñoz, and M. D. Baró, Phys. Rep. 422, 65 (2005).

${ }^{14}$ J. Nogués and I. K. Schuller, J. Magn. Magn. Mater. 192, 203 (1999).

${ }^{15}$ A. E. Berkowitz and K. Takano, J. Magn. Magn. Mater. 200, 552 (1999).

${ }^{16}$ R. L. Stamps, J. Phys. D 33, R247 (2000).

${ }^{17}$ M. Kiwi, J. Magn. Magn. Mater. 234, 584 (2001).

${ }^{18}$ J. C. Wu, H. W. Huang, C. H. Lai, and T. H. Wu, J. Appl. Phys. 87, 4948 (2000).

${ }^{19}$ J. Yu, A. D. Kent, and S. S. P. Parkin, J. Appl. Phys. 87, 5049 (2000).

${ }^{20}$ J. Sort, A. Hoffmann, S. H. Chung, K. S. Buchanan, M. Grimsditch, M. D. Baró, B. Dieny, and J. Nogués, Phys. Rev. Lett. 95, 067201 (2005).

${ }^{21}$ Z.-P. Li, O. Petracic, J. Eisenmenger, and I. K. Schuller, Appl. Phys. Lett. 94, 057203 (2005).

${ }^{22}$ W. Jung, F. J. Castaño, D. Morecroft, C. A. Ross, R. Menon, and H. I. Smith, J. Appl. Phys. 97, 10K103 (2005).

${ }^{23}$ Z. B. Guo, Y. K. Zheng, K. B. Li, Z. Y. Liu, P. Lou, Y. T. Shen, and Y. H. Wu, J. Appl. Phys. 93, 7435 (2003).

${ }^{24}$ R. Nakatani, T. Yoshida, Y. Endo, Y. Kawamura, M. Yamamoto, T. Takenaga, S. Aya, T. Kuroiwa, S. Beysen, and H. Kobayashi, J. Magn. Magn. Mater. 286, 31 (2005).

${ }^{25}$ V. Baltz, J. Sort, S. Landis, B. Rodmacq, and B. Dieny, Phys. Rev. Lett. 94, 117201 (2005).

${ }^{26}$ S. Soeya, T. Imagawa, K. Mitsuoka, and S. Narishige, J. Appl. Phys. 76, 5356 (1994).

${ }^{27}$ S. Brück, J. Sort, V. Baltz, S. Suriñach, J. S. Muñoz, B. Dieny, M. D. Baró, and J. Nogués, Adv. Mater. (Weinheim, Ger.) 17, 2978 (2005). 